\title{
THE FIRST VARIATION OF AN INDEFINITE WIENER INTEGRAL
}

\author{
ROBERT H. CAMERON
}

1. Introduction. It is the purpose of this paper to obtain a formula for $\delta G$ when $G$ is the indefinite Wiener integral

$$
G(u)=\int_{x(t) \leqq u(t)}^{W} F(x) d_{W} x .
$$

Here $x$ is understood to be a variable point in the Wiener space $C$ of continuous functions $x(t)$ defined on $0 \leqq t \leqq 1$ and vanishing at $t=0$. The integration is performed over the set $S_{u}$ of elements $x(t)$ of $C$ which satisfy for all $t$ the inequality

$$
x(t) \leqq u(t) .
$$

The function $u(t)$ need not be a member of $C$, but can be any Borel measurable function defined on $0 \leqq t \leqq 1$, and may even be permitted to take on infinite values.

The Wiener integral of a functional is simply the Lebesgue integral of the functional with respect to Wiener's measure $[4]^{1}$ in $C$. This measure is not invariant under translations, but is in other respects a Lebesgue measure based on intervals of the form

I:

$$
\alpha_{i}<x\left(t_{j}\right)<\beta_{j}
$$

$$
\text { (where } 0<t_{1}<t_{2}<\cdots<t_{n} \leqq 1 \text { ), }
$$

having the measure

$$
\begin{array}{r}
m_{W}(I)=\frac{1}{\left(\pi^{n} t_{1}\left(t_{2}-t_{1}\right) \cdots\left(t_{n}-t_{n-1}\right)\right)^{1 / 2}} \int_{\alpha_{n}}^{\beta_{n}} \cdots \int_{\alpha_{1}}^{\beta_{1}} \exp \left\{-\frac{\zeta_{1}^{2}}{t_{1}}\right. \\
\left.-\frac{\left(\zeta_{2}-\zeta_{1}\right)^{2}}{t_{2}-t_{1}}-\cdots-\frac{\left(\zeta_{n}-\zeta_{n-1}\right)^{2}}{t_{n}-t_{n-1}}\right\} d \zeta_{1} \cdots d \zeta_{n} .
\end{array}
$$

We denote the Wiener integral of a measurable functional $F(x)$ over a measurable set $S \subset C$ by

$$
\int_{S}^{W} F(x) d_{W} x .
$$

In case $S$ is not contained in $C$ but $S C$ is measurable, we define

Presented to the Society, November 26, 1949; received by the editors November 30, 1950.

${ }^{1}$ Numbers in brackets refer to the bibliography at the end of the paper. 


$$
m_{W}(S)=m_{W}(S C) \text { and } \int_{S}^{W} F(x) d_{W} x=\int_{S C}^{W} F(x) d_{W} x .
$$

In addition to finding $\delta G$ when $G$ is given by (1), we shall also find certain transformation formulas for Wiener integrals taken over the whole of $C$. In particular, we shall obtain what may be considered as a formula for integration by parts in function space.

Finally, we show that these formulas may be used to evaluate certain Wiener integrals. As an example we show that

$$
\int_{C}^{W}\left[\log \int_{0}^{1} \alpha(t) e^{x(t)} d t\right] x(s) d_{W} x=\frac{s}{2}
$$

when $0<s<1$, if $\alpha(t)$ is non-negative and of class $L_{1}$ and not equivalent to zero on $s \leqq t \leqq 1$.

\section{Sub-summable functionals.}

DeFinitions. Let $f(u)$ be a real or complex function defined on a set $S$ of an abstract space in which a measure is defined. Then $f(u)$ will be called "sub-summable" on $S$ if there exists a function $g(u)$ which is summable on a measurable set $\mathcal{S}$ containing $S$ and satisfies on $S$ the inequality $|f(u)| \leqq g(u)$.

It is clear that if $f(u)$ is also measurable on $S$, then it is summable on $S$ (and, of course, $S$ is also measurable).

LEMMA. Let $y_{0}(t) \in C$ be absolutely continuous and have a derivative $y_{0}^{\prime}(t)$ which is essentially of bounded variation ${ }^{2}$ on $[0,1]$; let $S$ be $a$ Wiener measurable subset of $C$; and for each positive $\lambda$ let $S^{\lambda}$ be the set of all $x(t)$ of the form $u(t)+h y_{0}(t)$, where $u \in S$ and $|h| \leqq \lambda$. Let $\epsilon>0$, $\eta>0$ and let $F(x)$ be a functional defined on $S^{\text {et }}$ such that

$$
\sup _{|h| \leqq \eta}\left|F\left(x+h y_{0}\right)\right|
$$

is sub-summable on $S^{e}$. Then if $P(\omega)$ is any polynomial, it follows that there exists $\eta_{1}>0$ for which

$$
\begin{aligned}
& \sup _{|h| \leqq \eta_{1}}\left|F\left(x+h y_{0}\right)\right| \\
& \cdot \exp \left\{2 \eta_{1}\left|\int_{0}^{1} y_{0}^{\prime}(t) d x(t)\right|\right\} P\left(\int_{0}^{1} y_{0}^{\prime}(t) d x(t)\right)
\end{aligned}
$$

is sub-summable on $\mathrm{S}$.

2 Here and elsewhere in this paper the requirement that a function be "essertially of bounded variation" can be replaced by the requirement that it be "of vlass $L_{2}$ " if Stieltjes integrals are interpreted as Paley-Wiener-Zygmund integrals $[3,1]$. 
For the proof, choose $\eta_{2}>0$ so that $\eta_{2}<\epsilon$ and $\eta_{2}<\eta / 2$, and apply the translation theorem $[2 ; 1]$ to a functional $G(x)$ which is summable on a measurable set containing $\mathcal{S}^{\boldsymbol{\epsilon}}$ and which satisfies on $\mathcal{S}^{\boldsymbol{\epsilon}}$

$$
G(x) \geqq \sup _{|h| \leqq \eta}\left|F\left(x+h y_{0}\right)\right| .
$$

We translate by $\eta_{2} y_{0}$ and also by $-\eta_{2} y_{0}$, and obtain

$$
\begin{aligned}
\int_{\left(x \mp \eta_{2} y_{0}\right) \in S}^{W} G(x) d_{W} x=\exp \left\{-\eta_{2}^{2} \int_{0}^{1}\left[y_{0}^{\prime}(t)\right]^{2} d x(t)\right\} \\
\cdot \int_{S}^{W} G\left(x \pm \eta_{2} y_{0}\right) \exp \left\{\mp 2 \eta_{2} \int_{0}^{1} y_{0}^{\prime}(t) d x(t)\right\} d_{W} x .
\end{aligned}
$$

Here the existence of the first member follows from the fact that we are integrating over a measurable subset of $\mathcal{S}^{\epsilon}$, and the existence of the second follows from that of the first by the translation theorem. Moreover, since $2 \eta_{2}<\eta$, we have by (5) for $x \in S$ and for both upper and lower signs,

$$
\sup _{|h| \leqq \eta_{2}}\left|F\left(x+h y_{0}\right)\right| \leqq G\left(x \pm \eta_{2} y_{0}\right),
$$

and hence it follows from the existence of the Wiener integral in the second member of (6) that

$$
\sup _{|h| \leqq \eta_{2}}\left|F\left(x+h y_{0}\right)\right| \exp \left\{ \pm 2 \eta_{2} \int_{0}^{1} y_{0}^{\prime}(t) d x(t)\right\}
$$

is sub-summable on $S$. Thus the maximum of these two functionals is also sub-summable; that is,

$$
\sup _{|h| \leqq \eta_{2}}\left|F\left(x+h y_{0}\right)\right| \exp \left\{2 \eta_{2}\left|\int_{0}^{1} y_{0}^{\prime}(t) d x(t)\right|\right\}
$$

is sub-summable on $S$. The sub-summability of (4) on $S$ follows immediately for positive $\eta_{1}<\eta_{2}$, and hence the lemma is established.

\section{The first variation of a Wiener integral and vice versa.}

THEOREM I. Let $y_{0}(t) \in C$ be absolutely continuous and have a derivative $y_{0}^{\prime}(t)$ which is essentially of bounded variation ${ }^{2}$ on $0 \leqq t \leqq 1$, and let $F(x)$ be a Wiener summable functional over the set $S_{u_{0}+2 \epsilon}$, where $u_{0}(t)$ is Borel measurable on $0 \leqq t \leqq 1$ (and may even be infinite there), $\epsilon>0$, and

$\delta_{u}$ :

$x(t) \leqq u(t)$,

$0 \leqq t \leqq 1 ; x \in C$ 
Let $F(x)$ have a first variation

$$
\left.\delta F \equiv \delta F\left(x \mid y_{0}\right)=\frac{d}{d h} F\left(x+h y_{0}\right)\right]_{h=0}
$$

for all $x \in \mathcal{S}_{u_{0+26}}$. Then if $0<\eta \max \operatorname{mat}_{01}\left|y_{0}(t)\right| \leqq \epsilon$ and

$$
\sup _{|h| \leqq \eta}\left|\delta F\left(x+h y_{0} \mid y_{0}\right)\right|
$$

is Wiener sub-summable in $x$ on $S_{u_{0}+\epsilon}$, it follows that the functional

$$
G(u)=\int_{x(t) \leqq u(t)}^{W} F(x) d_{W} x
$$

has a first variation

$$
\left.\delta G=\delta G\left(u \mid y_{0}\right)=\frac{d}{d h} G\left(u+h y_{0}\right)\right]_{h=0}
$$

whenever $u(t) \leqq u_{0}(t)$ on $0 \leqq t \leqq 1$ and $u$ is Borel measurable. Moreover the value of the variation is given by the following integrals (which necessarily exist):

$$
\begin{aligned}
\delta G\left(u \mid y_{0}\right)= & \int_{x(t) \leqq u(t)}^{W} \delta F\left(x \mid y_{0}\right) d_{W} x \\
& -2 \int_{x(t) \leqq u(t)}^{W} F(x)\left[\int_{0}^{1} y_{0}^{\prime}(t) d x(t)\right] d_{W} x .
\end{aligned}
$$

For the proof, we note that if $x+h y_{0} \in \mathcal{S}_{u_{0}+2 e}$,

$$
\begin{aligned}
\delta F\left(x+h y_{0} \mid y_{0}\right) & \left.=\frac{d}{d \lambda} F\left(x+h y_{0}+\lambda y_{0}\right)\right]_{\lambda=0} \\
& \left.=\frac{d}{d \mu} F\left(x+\mu y_{0}\right)\right]_{\mu=h}=\frac{d}{d h} F\left(x+h y_{0}\right) ;
\end{aligned}
$$

and since the first member of this equation exists, so does the last.

Again, it is clear that $S_{u+2 \epsilon}$ is convex, so that if $x \in S_{u+2 e}$ and $x+h y_{0} \in \mathcal{S}_{u+2 \epsilon}$, we have $x+\theta h y_{0} \in \mathcal{S}_{u+2 \epsilon}$ for all $\theta$ in $0 \leqq \theta \leqq 1$. Thus by the law of the mean we obtain $F\left(x+h y_{0}\right)=F(x)+h \delta F\left(x+\theta h y_{0} \mid y_{0}\right)$ for some $\theta$ in $0<\theta<1$ depending on $h$. Hence it follows from the subsummability of (8) and of $F(x)$ that

$$
\sup _{|h| \leqq \eta}\left|F\left(x+h y_{0}\right)\right|
$$

is sub-summable on $\boldsymbol{S}_{u_{0}+\bullet}$. 
Now for $|h| \leqq \eta$ and $u$ a Borel measurable function satisfying $u(t) \leqq u_{0}(t)$, we have by the translation theorem (which guarantees the existence of the last member)

$$
\begin{aligned}
& G\left(u+h y_{0}\right)=\int_{x(t)-h y_{0}(t) \in \mathcal{S}_{u}}^{W} F(x) d_{W} x \\
&= \exp \left\{-h^{2} \int_{0}^{1}\left[y_{0}^{\prime}(t)\right]^{2} d t\right\} \int_{\mathcal{S}_{u}}^{W} F\left(x+h y_{0}\right) \\
& \cdot \exp \left[-2 h \int_{0}^{1} y_{0}^{\prime}(t) d x(t)\right] d_{W} x .
\end{aligned}
$$

Differentiating formally with respect to $h$ and then setting $h=0$, we obtain

$$
\begin{aligned}
\delta G\left(u \mid y_{0}\right)= & \left.\frac{d}{d h} G\left(u+h y_{0}\right)\right]_{h=0} \\
= & \int_{S_{\iota}}^{W}\left[\frac { d } { d h } \left\{F\left(x+h y_{0}\right)\right.\right. \\
= & \left.\left.\quad \exp \left[-2 h \int_{0}^{1} y_{0}^{\prime}(t) d x(t)\right]\right\}\right]_{h=0} d_{W} x \\
& -2 \int_{S_{S_{u}}}^{W} F(x)\left[\int_{0}^{1} y_{0}^{\prime}(t) d x(t)\right] d_{W} x .
\end{aligned}
$$

To justify this differentiation under the integral sign (and incidentally show that all members of (13) exist), we must show that the differentiated integrand is dominated for small $h$ by a summable functional; that is, we must show that

$$
\begin{aligned}
\sup _{|h| \leq \eta_{1}} \mid\left\{\delta F\left(x+h y_{0} \mid y_{0}\right)-2 F\left(x+h y_{0}\right)\right. & \left.\int_{0}^{1} y_{0}^{\prime}(t) d x(t)\right\} \\
\cdot \exp & \left\{-2 h \int_{0}^{1} y_{0}^{\prime}(t) d x(t)\right\} \mid
\end{aligned}
$$

is sub-summable on $S_{u}$ for some $\eta_{1}>0$. But it follows from the subsummability of (8) on $S_{u_{0}+c}$ and the lemma that for some $\eta_{2}>0$

$$
\sup _{|n| \leq \eta_{2}}\left|\delta F\left(x+h y_{0} \mid y_{0}\right)\right| \exp \left\{2 \eta_{2}\left|\int_{0}^{1} y_{0}^{\prime}(t) d x(t)\right|\right\}
$$


is sub-summable on $S_{u_{0}}$. Similarly it follows from the sub-summability of (12) on $S_{u_{0}+e}$ and the lemma that for some $\eta_{3}>0$,

$$
\sup _{|h| \leq \eta_{3}}\left|F\left(x+h y_{0}\right)\right| \exp \left\{2 \eta_{3}\left|\int_{0}^{1} y_{0}^{\prime}(t) d x(t)\right|\right\}\left|\int_{0}^{1} y_{0}^{\prime}(t) d x(t)\right|
$$

is sub-summable on $\delta_{u_{0}}$. Taking $\eta_{1}=\min \left(\eta_{2}, \eta_{3}\right)$, we obtain the subsummability of (14) on $S_{u} \subset S_{u_{0}}$ and hence the justification of (13), including the existence of all its members. Thus the theorem is established.

An important special case of Theorem 1 is obtained if $u(t) \equiv u_{0}(t)$ $\equiv+\infty$, so that we integrate over the whole space $C$. In this case also $u(t)+h y_{0}(t) \equiv+\infty$ and $G\left(u+h y_{0}\right)$ is constant and $\delta G\left(u \mid y_{0}\right)=0$. We state the result as a separate theorem.

THEOREM II. Let $y_{0}(t)$ be absolutely continuous and have a derivative $y_{0}^{\prime}(t)$ which is essentially of bounded variation ${ }^{2}$ on $0 \leqq t \leqq 1$, and let $F(x)$ be a Wiener summable functional over $C$. Let $F(x)$ have a first variation $\delta F=\delta F\left(x \mid y_{0}\right)$ for all $x \in C$ such that

$$
\sup _{|h| \leq \eta}\left|\delta F\left(x+h y_{0} \mid y_{0}\right)\right|
$$

is Wiener summable in $x$ on $C$ for some $\eta>0$. Then it follows that

$$
\int_{C}^{W} \delta F\left(x \mid y_{0}\right) d_{W} x=2 \int_{C}^{W} F(x)\left[\int_{0}^{1} y_{0}^{\prime}(t) d x(t)\right] d_{W} x .
$$

As a corollary to Theorem II we obtain a formula for "integration by parts in function space." We replace $F(x)$ by $F(x) G(x)$.

CoROllary. Let $y_{0}(t)$ be absolutely continuous and have a derivative $y_{0}^{\prime}(t)$ which is essentially of bounded variation ${ }^{2}$ on $0 \leqq t \leqq 1$, and let $F(x)$ and $G(x)$ be Wiener measurable functionals on $C$ such that $F(x) G(x)$ is Wiener summable on $C$. Let $F$ and $G$ have first variations $\delta F$ and $\delta G$ such that

$$
G(x) \sup _{|h| \leqq \eta}\left|\delta F\left(x+h y_{0} \mid y_{0}\right)\right| \text { and } F(x) \sup _{|h| \leqq \eta}\left|\delta G\left(x+h y_{0} \mid y_{0}\right)\right|
$$

are Wiener summable in $x$ on $C$ for some $\eta>0$. Then it follows that

$$
\begin{aligned}
\int_{C}^{W} F(x) \delta G\left(x \mid y_{0}\right) & d_{W} x \\
& =\int_{C}^{W} G(x)\left[2 F(x) \int_{0}^{1} y_{0}^{\prime}(t) d x(t)-\delta F\left(x \mid y_{0}\right)\right] d d_{W} .
\end{aligned}
$$




\section{The Wiener integral of a Volterra derivative.}

Theorem III. Let $F(x)$ be a Wiener summable functional such that $F(x) \max _{0 \leqq t \leqq 1}|x(t)|$ is also Wiener summable, and such that the first variation

$$
\left.\delta F=\delta F(x \mid y)=\frac{d}{d h} F(x+h y)\right]_{h=0}
$$

exists for all $x$ and $y$ in $C$ and is expressible in the form

$$
\delta F(x \mid y)=\int_{0}^{1} F^{\prime}(x \mid t) y(t) d t
$$

where $F^{\prime}(x \mid t)$ is measurable in the product space of $x$ and $t$ as well as summable in $t$ for each $x$. (It is clear that $F^{\prime}(x \mid t)$ is the Volterra derivative of $F(x)$ at each point $(x, t)$ of the product space for which $F^{\prime}(x \mid t)$ is continuous in $(x, t)$.) Assume also that for each $y(t) \in C$ there exists a corresponding number $\eta=\eta(y)>0$ such that

$$
\sup _{|h| \leqq \eta, 0 \leqq t \leqq 1}\left|F^{\prime}(x+h y \mid t)\right|
$$

is Wiener summable in $x$ on $C$. Then it follows that $\int_{C}^{W} F(x) x(t) d_{W} x$ has an absolutely continuous derivative with respect to $t$ for $0 \leqq t \leqq 1$, and this derivative vanishes at $t=1$. Moreover

$$
\int_{C}^{W} F^{\prime}(x \mid t) d_{W} x=-2 \frac{d^{2}}{d t^{2}} \int_{C}^{W} F(x) x(t) d_{W} x
$$

for almost all $t$ on $0 \leqq t \leqq 1$, and, in particular, for each $t$ for which the left member is continuous. Specifically, (19) holds for each $t$ for which $F^{\prime}(x \mid t)$ is continuous in $t$ for almost all $x$ in $C$.

We shall prove that this theorem holds even when we weaken the hypotheses (16), (17), (18) by assuming that they hold not for all $y$ in $C$, but only for a sequence of values of $y$, namely $y=y_{n}$ $(n=1,2, \cdots)$, where each $y_{n}(t)$ has an absolutely continuous derivative $y_{n}^{\prime}(t)$ and satisfies $y_{n}(0)=y_{n}^{\prime}(1)=0$, and where the set of second derivatives $\left\{y_{n}^{\prime \prime}(t)\right\}$ is closed in $L_{2}$ on $0 \leqq t \leqq 1$. Then if $\|y\|$ $=\max _{0 \leqq t \leqq 1}|y(t)|$, we have by (17) for each $n=1,2, \cdots$ the inequality

$$
\sup _{|h| \leqq \eta_{n}}\left|\delta F\left(x+h y_{n} \mid y_{n}\right)\right| \leqq\left\|y_{n}\right\| \cdot \sup _{|h| \leqq \eta_{n}, 0 \leqq t \leqq 1}\left|F^{\prime}\left(x+h y_{n} \mid t\right)\right|,
$$

where $\eta_{n}$ denotes $\eta\left(y_{n}\right)$. Thus for each $n$, (18) and (20) imply that 
the hypotheses of Theorem II hold with $y_{n}$ replacing $y_{0}$, and we have from (15),

$$
\int_{C}^{W} \delta F\left(x \mid y_{n}\right) d_{W} x=2 \int_{C}^{W} F(x)\left[\int_{0}^{1} y_{n}^{\prime}(t) d x(t)\right] d_{W} x .
$$

Integrating by parts in the right member, remembering that $x(0)$ $=y_{n}^{\prime}(1)=0$, and using (17) in the left member, we obtain

$$
\begin{aligned}
\int_{C}^{W}\left[\int_{0}^{1} F^{\prime}(x \mid t) y_{n}(t) d t\right] & d W x \\
= & -2 \int_{C}^{W} F(x)\left[\int_{0}^{1} x(t) y_{n}^{\prime \prime}(t) d t\right] d_{W} x .
\end{aligned}
$$

Since by hypothesis $F(x) \cdot\|x\|$ is summable, we may apply the Fubini theorem to the right member, and since (18) is summable we may apply it to the left member. Thus

$$
\int_{0}^{1} y_{n}(t) \Psi(t) d t=-2 \int_{0}^{1} y_{n}^{\prime \prime}(t) d t \int_{C}^{W} F(x) x(t) d_{W} x
$$

where

$$
\Psi(t)=\int_{C}^{W} F^{\prime}(x \mid t) d_{W} x .
$$

We next integrate the left member of (21) by parts twice, and to simplify the notation we introduce the function

$$
\phi(t)=\int_{0}^{t} d u \int_{1}^{u} d s \int_{C}^{W} F^{\prime}(x \mid s) d_{W} x
$$

which obviously satisfies the conditions

$$
\begin{aligned}
& \phi(t) \text { and } \phi^{\prime}(t) \text { absolutely continuous on } 0 \leqq t \leqq 1, \\
& \phi^{\prime \prime}(t)=\Psi(t) \text { almost everywhere on } 0 \leqq t \leqq 1, \\
& \phi(0)=\phi^{\prime}(1)=0 .
\end{aligned}
$$

Thus we obtain from (21) by two integrations by parts, using $y_{n}(0)$ $=y_{n}^{\prime}(1)=0$ and $(23)$,

$$
\int_{0}^{1} y_{n}^{\prime \prime}(t)\left[\phi(t)+2 \int_{C}^{W} F(x) x(t) d_{W} x\right] d t=0 .
$$

But the $y_{n}^{\prime \prime}(t)$ are closed (and hence complete) in $L_{2}(0,1)$, and therefore (24) implies that for almost all $t$ on $0 \leqq t \leqq 1$ 


$$
\phi(t)=-2 \int_{C}^{W} F(x) x(t) d_{W} x .
$$

Actually, (25) is true for all $t$ on the unit interval, since both sides are continuous. The continuity of the right member follows from the continuity of $x(t)$ and the summability of $F(x)\|x\|$. Differentiating (25), we obtain for all $t$ on $0 \leqq t \leqq 1$,

$$
\phi^{\prime}(t)=-2 \frac{d}{d t} \int_{C}^{W} F(x) x(t) d_{W} x .
$$

From this and (23) it is clear that $\int_{C}^{W} F(x) x(t) d_{W} x$ has an absolutely continuous derivative on $0 \leqq t \leqq 1$ which vanishes at $t=1$. Another differentiation gives (19) for almost all $t$, and in particular whenever the left member is continuous. This must occur in view of (18) whenever $F^{\prime}(x \mid t)$ is continuous in $t$ for almost all $x$, and hence the theorem is established.

CoRollary. Theorem III holds when hypotheses (16), (17), (18) are assumed to hold only for a sequence of $y(t),\left\{y_{n}(t)\right\}$, such that each $y_{n}$ has an absolutely continuous derivative and $y_{n}(0)=y_{n}^{\prime}(1)=0$ and the second derivatives $\left\{y_{n}^{\prime \prime}(t)\right\}$ are closed in $L_{2}(0,1)$.

ExAmple. We conclude this paper by giving an example to show how Theorem II can be used to evaluate new Wiener integrals.

As our example, let

$$
F(x)=\log \left[\int_{0}^{1} \alpha(t) \exp \left\{\frac{x(t)}{\beta(t)}\right\} d t\right],
$$

where the integrand is understood to vanish when $\alpha(t)$ vanishes whether the exponential exists or not, and where $\alpha(t)$ and $\beta(t)$ satisfy the following conditions. We assume $\alpha(t) \in L_{1}, \alpha(t) \geqq 0$ on $0 \leqq t \leqq 1$, $\alpha(t)>0$ on a set of positive measure; $\beta(t) \in C$ and is absolutely continuous with a derivative essentially of bounded variation $;^{2}$ finally, we assume

$$
\int_{0}^{1} \alpha(t) \exp \left\{\frac{t}{4[\beta(t)]^{2}}\right\} d t<\infty ;
$$

where the integrand is (as above) interpreted to vanish when $\alpha(t)$ vanishes.

We first note that $F(x) \in L_{p}(C)$ for all positive $p$. For if $r>0$,

$$
|\log r|^{p}<p^{p} \max \left(r, r^{-1}\right)
$$


and hence

$$
\begin{aligned}
&|F(x)|^{p}<p^{p} \max \left[\int_{0}^{1} \alpha(t) \exp \left|\frac{x(t)}{\beta(t)}\right| d t,\right. \\
&\left.\left\{\int_{0}^{1} \alpha(t) \exp \left[-\left|\frac{x(t)}{\beta(t)}\right|\right] d t\right\}^{-1}\right] .
\end{aligned}
$$

Moreover by the Schwartz inequality

$$
\int_{0}^{1} \alpha(t) \exp \left\{-\left|\frac{x(t)}{\beta(t)}\right|\right\} d t \cdot \int_{0}^{1} \alpha(t) \exp \left|\frac{x(t)}{\beta(t)}\right| d t \geqq\left[\int_{0}^{1} \alpha(t) d t\right]^{2},
$$

so that

$$
|F(x)|^{p}<K \int_{0}^{1} \alpha(t) \exp \left|\frac{x(t)}{\beta(t)}\right| d t
$$

where

$$
K=p^{p} \max \left[1,\left(\int_{0}^{1} \alpha(t) d t\right)^{-2}\right]
$$

Thus

$$
\begin{aligned}
\int_{C}^{W}|F(x)|^{p} d_{W} x & <K \int_{0}^{1} \alpha(t) d t \int_{C}^{W} \exp \left|\frac{x(t)}{\beta(t)}\right| d_{W} x \\
& =\frac{K}{\pi^{1 / 2}} \int_{0}^{1} \frac{\alpha(t) d t}{t^{1 / 2}} \int_{-\infty}^{\infty} \exp \left[\left|\frac{s}{\beta(t)}\right|-\frac{s^{2}}{t}\right] d s \\
& <\frac{2 K}{\pi^{1 / 2}} \int_{0}^{1} \alpha(t) d t \int_{-\infty}^{\infty} \cosh \left(\frac{u t^{1 / 2}}{\beta(t)}\right) e^{-u^{2}} d u \\
& =2 K \int_{0}^{1} \alpha(t) \exp \left\{\frac{t}{4[\beta(t)]^{2}}\right\} d t \\
& <\infty .
\end{aligned}
$$

Now let $\mathcal{N}$ be the null set where $F(x)$ fails to exist, and let us define $F(x)$ to be zero on $\mathcal{N}$. We then have

$$
\begin{aligned}
F(x+h \beta) & = \begin{cases}F(x)+h & \text { when } x \in C-\mathcal{N}, \\
0 & \text { when } x \in \mathcal{N},\end{cases} \\
\delta F(x \mid \beta) & = \begin{cases}1 & \text { when } x \in C-\mathcal{N}, \\
0 & \text { when } x \in \mathcal{N} .\end{cases}
\end{aligned}
$$


Thus the hypotheses of Theorem II (with $y_{0}=\beta$ ) are satisfied and we obtain from (15)

$$
\int_{C}^{W}\left\{\log \left[\int_{0}^{1} \alpha(t) \exp \left(\frac{x(t)}{\beta(t)}\right) d t\right]\right\}\left[\int_{0}^{1} \beta^{\prime}(t) d x(t)\right] d_{W} x=\frac{1}{2} .
$$

In particular, if $\alpha(t)=0$ when $t<s$ and $\beta(t)=s^{-1} \min (s, t)$ for some fixed $s$ on $0<s<1$, we obtain formula (2) given in the introduction.

As another special case, take $\beta(t)=t / 2$ and $\alpha(t)=\phi(t) \exp \left(-t^{-1}\right)$, where $\phi(t)$ is non-negative and summable and not equivalent to zero on $0 \leqq t \leqq 1$. Clearly the required conditions on $\alpha$ and $\beta$ are satisfied, and we have

$$
\int_{C}^{W} x(1)\left[\log \int_{0}^{1} \phi(t) \exp \left[\frac{2 x(t)-1}{t}\right] d t\right] d_{W} x=1 .
$$

Other interesting formulas can be obtained by using the formula for "integrating by parts in function space."

\section{BIBLIOGRAPHY}

1. R. H. Cameron and Ross Graves, Additive functionals on a space of continuous functions. I, Trans. Amer. Math. Soc. vol. 70 (1951) pp. 160-176.

2. R. H. Cameron and W. T. Martin, Transformation of Wiener integrals under translations, Ann. of Math. vol. 45 (1944) pp. 386-396.

3. R. E. A. C. Paley, N. Wiener, and A. Zygmund, Notes on random functions, Math. Zeit. vol. 37 (1933) pp. 647-668.

4. N. Wiener, Generalized harmonic analysis, Acta Math. vol. 55 (1930) pp. 117-258.

UNIVERSITY OF Minnesota 